\title{
L'Humanisme trahi: sur un livre récent de Stéphane Toussaint
}

\section{Myriam Jacquemier}

\section{(2) OpenEdition}

1 Journals

Édition électronique

URL : http://journals.openedition.org/studifrancesi/7797

DOI : ERREUR PDO dans /localdata/www-bin/Core/Core/Db/Db.class.php L.34 : SQLSTATE[HY000]

[2006] MySQL server has gone away

ISSN : 2421-5856

Éditeur

Rosenberg \& Sellier

Édition imprimée

Date de publication : 1 juillet 2009

Pagination : 315-324

ISSN : 0039-2944

\section{Référence électronique}

Myriam Jacquemier, «L'Humanisme trahi: sur un livre récent de Stéphane Toussaint », Studi Francesi [En ligne], 158 (LIII | II) | 2009, mis en ligne le 30 novembre 2015, consulté le 07 janvier 2021. URL

http://journals.openedition.org/studifrancesi/7797; DOI : https://doi.org/10.4000/studifrancesi.7797

\section{(c) (i) (9)}

Studi Francesi è distribuita con Licenza Creative Commons Attribuzione - Non commerciale - Non opere derivate 4.0 Internazionale. 


\section{L'Humanisme trahi: sur un livre récent de Stéphane Toussaint}

Il est des lectures qui tombent à point nommé, soit qu'elles résolvent dans l'instant un problème donné, soit qu'elles éclairent d'une lumière crue qui en accentue les aspérités les diverses facettes d'une actualité brûlante et en empêchent résolument l'occultation. L'écriture polémique et engagée de l'ouvrage de Stéphane Toussaint, Humanismes et Antibumanismes, de Ficin à Heidegger ${ }^{l}$ qui a retenu toute mon attention, ne peut en effet être détachée du climat de crise sociétale qui hante nos médias depuis plusieurs mois et que les études humanistes ressentent plus que tout autres par leur sensibilité et l'importance de leur engagement autant intellectuel que politique et moral' ${ }^{2}$ Que nous invite à faire ce jeune chercheur, philosophe, spécialiste de l'humanisme ficinien, hormis à constater sans ménagement aucun, l'assassinat, programmé de longue date, de ce moment d'humanité hors du commun qui, sous l'égide de Cosme puis de Laurent de Médicis, accueillit sur les coteaux florentins, l'expression la plus universelle, la plus humaine et la plus douce d'une érudition ${ }^{3}$ toute consacrée au culte de l'homme dans ce qu'il a de meilleur, en son cœur, en son âme, en sa raison.

Deux motivations au cœur de mes préoccupations actuelles m'ont poussée à vouloir faire partager l'intérêt qu'a suscité la lecture de ce texte: d'abord, parce que, philosophiquement, il décortique, concept par concept, la rapide disparition, dans

(1) StÉPhane Toussaint, Humanismes et Antibumanismes, de Ficin à Heidegger, Paris, Les Belles Lettres, 2008. S. Toussaint est aussi l'auteur de Pic de la Mirandole, Commento, Les Formes de l'Invisible, Paris, L'Âge d'homme, 1989; L'Esprit du Quattrocento. Le De Ente et Uno de Pic de la Mirandole, Paris, Champion, 1995; De l'Enfer à la Coupole, Dante, Brunelleschi, Ficin, Rome, Bretschneider, 1997. Et plus spécifiquement, La dignità dell'uomo e la libertà della filosofia, in La filosofia del Rinascimento, éd. G. ERNST, Carocci, Rome, 2003, pp. 49-69; L'Ars de Marsile Ficin entre esthétique et magie, in L'Art de la Renaissance entre Science et Magie, Actes du Colloque international de Paris 20 22 juin 2002, éd. Ph. Morel, Rome-Paris, Somogy, 2006, pp. 453-467; Heidegger e l'bitlerismo in filosofia, in «Giornale critico della filosofia italiana», Anno LXXXV (LXXXVIII), Fasc. 3, settembredicembre 2006, pp. 522-530; et Kristeller e Garin: polemiche umanistiche. Nuovi documenti, in Platonismo, Neoplatonismo, Ermetismo fra Umanesimo e controriforma. XI Giornata L. Firpo, Convegno in onore di Cesare Vasoli, Torino 28-29 ottobre 2004, a cura A. E. BALDINI, Firenze, Olschki, sous presse. Enfin, S. Toussaint dirige la revue internationale de philosophie de la Renaissance «Accademia», dédiée à Ficin et au Néoplatonisme.

(2) Quand le livre a été pensé et écrit, la crise universitaire était manifestement connue; la crise financière n'avait laissé paraître que quelques «incidents» dont aucun expert ou si peu avaient annoncé la gravité. Depuis, en France comme en Italie, la question de la place des études du patrimoine culturel ancien (latin, langues et littérature médiévales et a fortiori littérature de la Renaissance) a été cruellement posée lors du projet ministériel de la refonte des programmes qui définissent le corpus de connaissances «nécessaire» à la professionnalisation des enseignants des premier et second degrés. Pourtant, les scientifiques poursuivent leur travail de mémoire. Les «Studi Francesi» ont récemment fait paraître un fascicule consacré à L'Humanisme en Europe et dédié à la mémoire d'un des professeurs qui en ont illustré la forme et l'esprit, le professeur Franco Simone («Studi francesi», n. 153, III-2007).

(3) Le mot français rend mal compte de l'idée originelle véhiculée par le terme d'eruditio en latin. S. Toussaint commente ce glissement p. 176, note 8. Un des mérites de l'étude du philosophe est de conserver la démarche philologique qui fut, à un moment donné, l'apanage d'un certain humanisme; le lecteur est ainsi conduit de la langue latine à la grecque, à l'allemande ou à la française, et pénètre dans les nuances notionnelles de chaque concept, gage d'une pensée précise et rigoureuse. 
l'histoire des idées, de cet héritage ficinien et pichien auquel je voue, comme l'auteur de cet ouvrage, un culte passionné ${ }^{4}$. Ensuite, parce que les années de décanat que je viens de vivre à la tête d'une faculté littéraire en France (2003-2008), m'ont conduite à cautionner, non sans contradiction douloureuse, les effets paradoxaux d'une réforme universitaire dont la deuxième partie du livre «Rentabilité» dissèque le moindre des facteurs à l'origine de ce renversement radical des valeurs. Il n'est plus possible, alors, de croire à un «accident» de l'histoire: la déshumanisation des programmes littéraires, jusqu'à la mise à mal de tout discours universitaire qui pourrait parler «de l'homme à l'homme» sans autre «profit» que «de le rendre meilleur» (les bumaniora $a^{5}$, s'intègre dans un processus dont on apprend qu'il se renforce de siècle en siècle et dont la supposée «modernité» n'est que la pâle métonymie d'une réalité plus agressive, la vanité pure et simple du concept actuel d'«bumanitas». D'ailleurs, la dialectique du texte fait rapidement coïncider les deux pans de la réflexion car le dévoiement progressif, sur un plan philosophique et idéologique, du contenu du concept d' «humanisme» suit (ou précède, telle est la question) les mutations socio-économiques, voire politiques des sociétés européennes jusqu'à n'être plus qu'un thème jugé obsolète et totalement abandonné à une recherche déconsidérée et isolée. La crise financière qui secoue le monde ne serait ainsi qu'une des manifestations dont la «crise de l'humanisme et des humanités» serait «le point culminant [...] désormais atteint» ${ }^{6}$, selon le constat sans appel qui est annoncé dès les premières pages du livre. En effet, qui, à l'évocation, banalisée aujourd'hui, du concept d'«humanisme» se souvient encore $\mathrm{du}$ «miracle» du cercle florentin incarné par Ficin et Pic, entre autres, et de son rayonnement sur les textes français de la Renaissance? Comment enseigner Scève, Ronsard voire Rabelais, Érasme, Montaigne à l'université quand tout le substrat conceptuel sur lequel se développe le champ du mot «bumanitas» est vidé de cet ancrage historique dont l'ouvrage de S. Toussaint démontre l'exemplarité? Reste-t-il encore une place dans l'éducation actuelle, pour l'enseignement d'une littérature qui ranime et actualise «une vision métaphysique de l'homme [...] qui s'ouvre à une forme de lutte universelle pour la valeur humaine sub specie hominis parmi les hommes et dans l'histoire»? ? N'est-ce pas au moment où la mondialisation estompe les contours d'un réel individualisé et où notre perception du temps s'affole qu'il s'avère utile de remettre en pleine lumière ce moment exceptionnel, sous l'égide des Médicis, que fut l'humanisme florentin et son prolongement francisant, «polarité fondamentale mise en place par la Renaissance même entre l'Antiquité et la modernité» telle que la définit le rapport Kristeller-Garin ${ }^{8}$ ?

L'éclosion d'une telle «école de pensée» s'inscrit en effet dans ce moment de l'histoire (après la Chute de Constantinople puis l'expulsion des Juifs d'Espagne) qui permet que se rencontrent au cœur de l'Europe d'alors, les héritages simultanés

(4) Mes propres recherches sur la kabbale chrétienne et tout particulièrement sur le thème de l'harmonie musicale du monde à la Renaissance m'ont permis à maintes reprises de m'enrichir des travaux de l'auteur. Un des textes qui témoigne le plus de cet héritage philosophique néoplatonicien et de son rayonnement dans la littérature française du XVI ${ }^{\mathrm{e}}$ siècle est le vaste Hymne en trois Cantiques écrit par un moine franciscain vénitien en 1525 puis traduit en langue française par Guy Le Fèvre de la Boderie en 1578 sous le titre L'Harmonie du Monde divisée en trois cantiques, oeuvre singulier et plein d'admirable erudition: Premierement composé en Latin par Francois Georges
Venitien E depuis traduict E illustré par Guy Le Fèvre de la Boderie secretaire de Monseigneur Frere unique du Roy, à Paris, chez Jean Macé, 1578. Francisci Georgi Veneti, Minoritanae familiae De Harmonia mundi totius cantica tria, Venetiis, in aedibus Bernardini de Vitalibus calcographi, An. D. 1525 (reéd. 1526), puis réimprimé à Paris en $1545,1546$.

(5) S. ToussainT, op. cit., p. 14. L'auteur évoque ces disciplines dites Humaniora considérées comme telles «parce qu'elles nous rendent plus humains».

(6) S. Toussaint, op. cit., p. 11.

(7) S. Toussaint, op cit., p. 18.

(8) S. Toussaint, op. cit., p. 19. 
de la Grèce antique qui valorise la philanthropia et idéalise la paidea, de l'bumanitas latin qui prône les principes d'érudition mais aussi d'urbanité comme condition de libération de l'esprit humain, et auxquels se mêlent la richesse de la scolastique, de la patristique et des cultures d'Orient (islamisme et arabisation enrichie de l'héritage chaldéen, égyptien et des sources hermétiques). La ressemblance avec notre «mondialisation» des savoirs pourrait nous inspirer si la force structurante, sur le plan psychologique, moral, politique et métaphysique, des «valeurs» humanistes (des vertus dirait Plotin) nous était connue. Car l'un des mérites du livre est de rappeler l'originalité de l'intégration des cultures dans le savoir re-constitué des humanistes florentins. Creuset d'une pensée nourrie de tous les horizons, l'humanisme du Quattrocento sut s'en inspirer sans en devenir esclave. Aucun des systèmes gréco-latins n'avait proposé dans leur complexité la réunion nécessaire et suffisante des «studia bumanitatis», de la «charitas» et de l'«unitas hominum», complexité qui structurait «une assise conceptuelle et doctrinale, embrassant une culture mondiale, de Zoroastre à Pléthon et de la Chaldée à Byzance» ${ }^{9}$ et que S. Toussaint pose comme la mesure absolue de laquelle s'éloignera, jusqu'à l'ignorer, l'évolution postérieure du concept. L'auteur insiste sur cette originalité du regard que porte l'humanisme florentin sur la complexité d'un monde dont il restructure les fondements. Ses disciples ne s'enferment pas dans une simple duplication du passé, aussi prestigieux soit-il: «En réinventant l'bumanitas antique, latine et grecque, les humanistes de la Renaissance instaurent effectivement une humanité autonome, non parce qu'elle s'estime séparée de Dieu ou de l'Être comme le soutiennent ses nombreux critiques depuis Maritain, mais dans la mesure où elle se révèle, aujourd'hui même, distincte des Anciens et incomprise des Modernes. Telle est l'autonomie historique et philosophique plus que théologique, de l'bumanitas du $\mathrm{XV}^{\mathrm{e}}$ siècle» ${ }^{10}$. Si l'enseignement en était diffusé, tel serait l'exemple que ces humanistes lettrés pourraient donner à notre époque en quête de repères: non pas copier une culture mais inventer un art de vivre associant l'harmonie du développement personnel, gage d'une individualisation respectée, à un principe de sociabilité fondé sur l'échange intellectuel et l'enrichissement raisonné auprès des cultures étrangères. S. Toussaint se plaît à rappeler ce «modèle, fort idéalisé par la suite, de symbiose intellectuelle, politique et sociale entre les idées, les arts et les hommes» ${ }^{11}$, échanges multidirectionnels qui permirent à ce qui fut une des premières académies des temps modernes de «s'approcher incontestablement d'un idéal vécu dans un idéal pensé» ${ }^{12}$. En insistant avec bonheur sur cet «idéal vécu» des Renaissants qui, s'opposant sans le nier au platonisme, relève le défi d'inscrire la notion d'bumanitas dans sa réalité charnelle, voire sensible, l'analyste humaniste redonne au mot «morale» sa valeur essentielle, celle d'aider l'humain à construire sa vie: «Après des siècles de pensée abstraite, les mots et les choses [devaient] se réconcilier. Cette civilisation de la parole donne intentionnellement au verbum le poids de la res» ${ }^{13}$. Il fait ainsi revivre un «style

(9) S. ToussainT, op. cit., p. 48.

(10) S. ToussainT, op. cit., p. 45.

(11) S. Toussaint, op. cit., p. 30.

(12) S. Toussaint, op. cit., p. 30. Pour ma part, j'ai antérieurement rapproché le modèle de Careggi de la création de la première Académie française créée par Charles IX en 1570 en montrant les enjeux psychologiques, politiques, moraux voire métaphysiques de cette création en plein acmé des guerres de religions. Cf. M. JACQUEMIER, L'influenza dell'Academia fiorentina nel progetto dell' Academia di musica e di poesia di Baïf, dans Rapporti e Scambi tra l'Umanesimo italiano e l'Umanesimo europeo, XI convegno internazionale di Chianciano-Pienza
19-22 luglio 1999, a cura di Luisa RoTONDI SECCHI Tarugi, Milano, Nuovi Orizzonti, 2001, pp. 65-84. Et du même auteur, Les Académies au XVI siècle, in Les Outils de la Connaissance, L'Enseignement et la Formation Intellectuelle en Europe de 1453 à 1715, sous la direction de Jean-Claude Colbus et Brigitte HÉBERT, Saint-Etienne, Publications de l'université de Saint-Etienne, 2006, pp. 221-238.

(13) S. Toussaint, op. cit., p. 51. J'ai analysé le même mécanisme appliqué à la conception de la musique en ce début de Renaissance française, musique autant métaphysique, cosmologique que sensible voire sensuelle; cf. M. JACQUEMIER, Musique et néoplatonisme dans "Les Amours" (1552) de Ronsard: 
de vie unique où éducation, sentiment et savoir sont indissociablement liés» ${ }^{14}$, rappel utile à nos contemporains soucieux de faire prévaloir le pragmatisme sur l'idéalité. Mais en l'occurrence, pas n'importe quel pragmatisme car toute la perfection du modèle réside précisément dans la thèse que défend l'auteur selon laquelle seul l'humanisme ficinien offrait cette caractéristique holistique qui définissait l'bumanitas dans toutes ses dimensions, douceur, humanité de l'homme, humanités littéraires, suscitant ainsi la création d'une rhétorique originale qui éclairera une grande partie de la littérature de la Renaissance française. La lettre à Minerbetti de Marsile Ficin que cite et traduit $\mathrm{S}$. Toussaint confirme cet aboutissement. Lorsque celui-ci déclare que «les hommes vivent frères dans le grand corps de l'humanité», se dessine un nouvel espace-temps, l'idée d'un «grand être humain [...] où les sapientes [...] sont Hermès, Orphée, Zoroastre, grands dépositaires de la sagesse poétique et des images symboliques du divin» ${ }^{15}$. La boucle est bouclée au sens où la quête d'une esthétique nouvelle peut servir la représentation d'une métaphysique de l'être conforme à celle dont rêve Pic, «projet d'une humanité magique, érotique et gracieuse» qui manque tant à la culture actuelle ${ }^{16}$.

C'est à partir des valeurs de référence du courant humaniste que S. Toussaint reconstitue l'entreprise de démolition du concept «bumanitas» telle qu'elle s'exerça au cours de l'histoire, du XVIII siècle à la fin du XX', et même jusqu'à aujourd'hui, pouvons-nous ajouter. L'analyse remarque comment «selon un mécanisme impitoyable, la philosophie glisse irrésistiblement de l'humanisme antique à l'antihumanisme moderne, adversaire déclaré de Descartes et de Voltaire» ${ }^{17}$, laissant dès le siècle des Lumières les bâtisseurs de l'anthropologie s'emparer, pour le défaire, du principe «triadique» ficinien au profit d'une lecture scientifique de la spécificité humaine ${ }^{18}$. La démarche argumentative du philosophe est logique: partant de cette composition tripartite qui, par sa complexité, conférait un équilibre parfait à ce modèle d'humanité, il choisit d'en poursuivre, au gré des époques concernées, le progressif démembrement. Parmi les trois composantes (philantbropia, eruditio, unitas bumana) la valeur «philanthropie» conserva au cours du processus analysé la plus grande prégnance; mais, en se maintenant de façon majoritaire dans la triade, elle occulta progressivement le principe d' «érudition», abandonnant à «l'humanisme des bons sentiments» ${ }^{19}$ la quasi-totalité de l'espace notionnel qui devait constituer, tout particulièrement pour

du mythe à la réalité, Actes du Colloque Ronsard, à propos des Amours, Journées du Centre Jacques de Laprade, Pau, novembre 1997, Biarritz, Atlantica, 1997 , p. 115-148. De même, La musique comme principe cosmologique, ou variations sur le thème du même et de l'autre, in Histoire et Littérature au siècle de Montaigne, Mélanges offert au Professeur Claude-Gilbert Dubois, Genève, Droz, 2001, pp. 379-400. Et Le thème de l'harmonie du mond: entre musique et sacré, de François Georges de Venise à Marin Mersenne, in Il Sacro nel Rinascimento, a cura da Luisa Rotondi Secchi TARUgi, Actes du colloque de Chianciano-Pienza, 17-20 juillet 2000, Firenze, Franco Cesati Editore, 2002, pp. 139-162.

(14) S. Toussaint, op. cit., p. 51.

(15) S. ToussainT, op cit., p. 56.

(16) S. Toussaint, op. cit., p. 44. Voir la rhétorique des Livres du monde qui avait pour mission esthétique de respecter la loi selon laquelle l'écriture doit être conforme à la hauteur du sujet considéré. Cf. M. JacQuemier, Livre parmi les livres: l'origi- nalité des Livres du Monde in L'Europa del Libro nell'Età dell'Umanesimo, 16-19 juillet 2002, Actes du colloque de Chianciano-Pienza, a cura da Luisa Rotondi Secchi Tarugi, Firenze, Franco Cesati Editore, 2004, pp. 663-684.

(17) S. Toussaint, op. cit., p. 59.

(18) S. ToussainT, op cit., p. 34: «On passe ainsi d'un concept théologique et juridique à une civilisation des mœurs puis à une théorie cosmopolite, enfin à une anthropologie rationnelle».

(19) S. ToussainT, op. cit., p. 67. En redonnant la parole à de nombreux néohumanistes ignorés en France, S. Toussaint rappelle comment les réformes pédagogiques souhaitées par Niethammer et d'autres ont d'emblée cherché à présenter, comme résistance suprême à l'industrialisation des masses, le développement harmonieux d'une culture classique conforme aux canons de l'antiquité. Ce fut à la fois un bien et un mal car le Quattrocento n'a pas ressuscité l'antiquité en tant que telle mais l'a réinventée, voir la note 10 supra. 
les philanthropinistes, la principale, puis peu à peu la seule valeur «humaine» reconnue comme fondement de l'humanité20. Entre temps, le naturalisme et son pendant épistémologique, l'anthropologie, accroissaient leur socle conceptuel et méthodologique, renforçant l'idée d'une possible science de l'homme qui, en en intégrant l'étude dans un cadre défini par l'extériorité naturelle et sociale, perdit quasi définitivement de vue le principe d'intériorité du Quattrocento qui en constituait le fondement ${ }^{21}$. Certes, la Renaissance ignorait l'industrie et la technologie qui lui était jointe, mais elle avait déjà structuré un ordre marchand qui faisait «cohabiter harmonieusement ses marchands-écrivains, ses banquiers-lettrés et ses Berufshumanisten, ses humanistes de métier, sans jamais montrer les signes d'une destruction de l'humanisme par lui-même» ${ }^{22}$.

Là réside sans doute toute la fragilité du deuxième pan de l'argumentation de Stéphane Toussaint. Il reconnaît lui-même qu'il ne cherche pas, ni même ne pense souhaitable un «retour au Quattrocento», bien évidemment. Mais il est révélateur que l'analyse consacrée à l'ennemi de l'intérieur (le néohumanisme), puis à celui de l'extérieur (l'antihumanisme) jusqu'à son déplacement dans l'espace structurel du concept (l'anthropologie conduite à conclure à son impuissance ${ }^{23}$ ) ne soit prise en compte qu'à partir de ce XVIII ${ }^{\mathrm{e}}$ siècle, au moment où l'Europe vit sa première grande mutation économique. Dès lors, comment comparer deux types de constructions mentales inscrits dans des contextes économico-politiques de principes si radicalement opposés? Cette remarque ne remet pas en cause la pertinence de l'ouvrage du philosophe puisque, au contraire, l'auteur démasque ainsi cette «usurpation» de titre qui fait s'appeler «humanisme» une succession de philosophies qui n'en sont qu'un pâle reflet si l'on maintient à l'humanisme ficinien la valeur de référence ${ }^{24}$. De plus, en passant des thèses des néohumanistes à celles des antihumanistes les plus radicaux et les plus contemporains, l'étude conserve une merveilleuse cohérence en retraçant les multiples avatars par lesquels l'homme put chercher à se «dire» lui-même, dans sa spécificité et selon les mérites de sa dignité quels qu'en soient les critères choisis. Ainsi, le discours est toujours ambivalent: tout en déplorant l'échec de toute métaphysique à pouvoir, encore, rendre compte de l'homme, S. Toussaint en dessine une image nostalgique qui, dans la période très précise que nous vivons, pourrait en raviver la flamme. En ce sens, l'auteur oppose certainement le pouvoir du symbole à celui du concept, en d'autres termes celui de l'art humain à celui de la théorie anthropologique. Là est bien l'enjeu: une métaphysique, et pas la moindre, pourrait-elle encore éclairer l'humain dans la recherche de son propre sens, ou la question elle-même est-elle définitivement impensable, du moins sans le secours d'une image humaine, fondée sur un corpus d'auteurs humanistes? Pour que l'humanisme du Quattrocento en soit le bénéficiaire, et non ces ésotérismes de bazar dont les gourous de tous bords propagent l'idéologie avec de plus en plus d'écho, encore faut-il que l'enseignement

(20) S. Toussaint, op. cit., p. 66. Évoquant les fractures philosophiques internes à l'bumanitas, l'auteur explique comment «l'humanisme des bons sentiments» put s'accompagner du mépris des lettrés «coupables de précipiter l'humanisme dans un dressage aristocratique». La version «humanitariste» de l'humanisme donna lieu au courant des «philanthropiniens», cf. S. Toussaint, op. cit., pp. 73-77.

(21) S. ToussainT, op. cit., p. 41: «L'Humanisme $\mathrm{du}$ Quattrocento florentin reste incompris pour deux motifs distincts que sont l'écroulement de l'érudition contemporaine et la conviction qu'une raison scientifique domine la grande leçon de l'homme moderne». Le processus est amorcé dès la fin du XVII ${ }^{\mathrm{e}}$ siècle, selon l'auteur.

(22) S. ToussainT, op. cit., p. 104 et note 97.

(23) S. Toussaint, op. cit., p. 61: «Et ainsi s'achève le périple étonnant d'une science comme l'anthropologie, partie pour expliquer la nature humaine mais finissant par expliquer pourquoi elle ne l'a pas trouvée».

(24) S. Toussaint, op. cit., p. 29. Le but est énoncé dès le début de l'ouvrage, à savoir, sortir des «mille acceptions» du mot «qui minent la précision intellectuelle [...] pour fixer dans les textes du Quattrocento un axe utile et certain, celui d'une réinvention intellectuelle de l'humain». 
de ces moments lumineux de l'histoire des idées que sont les textes du XVI ${ }^{e}$ siècle, entre autres, ne soit pas oublié, dénaturé, déconsidéré au prétexte fallacieux qu'il n'est pas exploitable par la rentabilité. Telle est la dramatique réalité qui se vit aujourd'hui de façon irrémédiable semble-t-il, sur les bancs de l'université européenne, parachevant le processus rentabiliste analysé de façon critique par l'auteur.

Car l'un des multiples intérêts de l'ouvrage est de rappeler que les modifications apportées par les divers courants humanistes (puis par les antihumanistes) au concept référant d'Humanitas, l'ont été sous la pression de bouleversements socioéconomiques, eux-mêmes promoteurs de réformes universitaires dont les dirigeants espéraient une «éducation» (ou une «formation» ${ }^{25}$ ) adaptée à leurs besoins. Ce fut le cas de Niethammer comme celui de Humboldt, mais aussi, plus récemment, ceux de Bourdieu ou de Derrida, produits de cette époque de mutation, sinon de révolution, dont ils ont finalement traduit l'aporie. Stéphane Toussaint rappelle à plusieurs reprises que les «néohumanistes ne couronnent pas l'idéal de l'humanité universelle raisonnable pour satisfaire en premier lieu leur tyrannique exigence abstraite [mais parce qu'] ils réagissent activement à la crise pédagogique du XVIII ${ }^{\mathrm{e}}$ siècle et à l'Industriepädagogik qui l'illustre» ${ }^{26}$. L'auteur précise: «On entend par ce mot le mouvement d'éducation au travail manuel fondé par Philip von Sextro, Arnold Wageman et quelques autres à partir de 1785 c'est-à-dire après la création d'une Industrieschule en 1779». La crise des humanités se résume à opposer les deux termes d'une alternative qui s'exprime par l'un ou l'autre principe: la société doit-elle suivre l'école, ou l'école la société ${ }^{27}$, alternative devant laquelle $\mathrm{S}$. Toussaint prône une intellectuelle révolte qui empêcherait «la déchéance irréversible de l'éducation en formation, de la formation en professionnalisation et de la professionnalisation en rentabilisation» ${ }^{28}$.

La seconde partie de l'ouvrage «Rentabilité» explique en effet, si cela nous avait échappé, à quel point circulaires ministérielles, nationales ou européennes, ouvrages didactiques ou philosophiques ont creusé de façon inéluctable, depuis plus de cinquante ans, la tombe des humanités classiques sous l'hégémonie d'abord des sciences, puis de la technique, à présent de l'industrie $e^{29}$. Mais sans doute, pour l'auteur, normalien et philosophe de la Renaissance, la pire des condamnations s'exerce-t-elle à l'encontre de ceux qui, nourris aux mêmes nourritures spirituelles que lui, ont trahi leur mission de «transmetteur» et sont devenus, volontairement ou non, les complices des économistes, gestionnaires, politiques des divers ministères qui ont fait pression sur les dirigeants de l'Éducation Nationale et de l'enseignement supérieur pour faire entrer, ce coup-ci de façon massive donc définitive, les réformes pédagogiques dans la sphère du quantifiable, de l'économique, du productif. Telle me paraît être la grande différence avec les étapes antérieures de ce même combat qui se joue depuis plus de trois siècles ${ }^{30}$. Aujourd'hui, le renversement est radical puisque la massification, ou

(25) S. Toussaint, op. cit., p. 72 et suivantes. L'auteur définit, par la sémantique des concepts allemands, les diverses nuances qui font peu à peu s'opposer, au sein même de l'humanisme, les diverses tendances du courant. Cfr. entre autres, la différence entre Beruf, à la fois «métier» mais aussi «vocation» et Bildung, la «formation».

(26) S. ToussainT, op. cit., p. 101.

(27) S. ToussainT, op. cit., p. 73: «De nos jours et dans la formation des étudiants, le modèle social, quel qu'il soit [...] a définitivement remplacé la science désintéressée [...]. Quant au Mensch [l'idéal normatif de l'homme raisonnable pensé par Kant] célébré par Kant, Niethammer, Evers, Jachmann, Humboldt et finalement par Jaeger, il finira par s'éclipser derrière les attentes d'une société pour laquelle l'humanisme équivaut tout au plus à l'humanitarisme».

(28) S. ToussainT, op. cit., p. 105.

(29) S. Toussaint, op. cit., p. 102.

(30) S. Toussaint, op. cit., p. 93: «L'affrontement contemporain du métier contre la vocation, de l'utilité contre la gratuité, de la production contre l'invention, toutes ces luttes condensées dans la mé- 
la démocratisation de l'enseignement supérieur ne laisse plus que peu d'espace pour ceux qui, contre vents et marées, désirent faire encore entendre la voix des «langues dites mortes» ou, plus près de nous, la langue rocailleuse de Rabelais ou la labyrinthique écriture de Montaigne. Pouvait-on l'éviter?

Nous devons y regarder de plus près, car si l'ensemble des problèmes et des explications soulevés et décortiqués sous le scalpel rigoureux de Stéphane Toussaint fonctionne à vif, l'expérience du terrain complexifie et nuance la logique des événements. J'ai, en tant que doyen pendant cinq ans, dû faire appliquer une grande partie des mesures décriées par l'auteur (recherche de l'efficacité immédiate, politique des résultats, objectifs quantifiés, rentabilité des enseignements), et tout en conservant le même attachement que lui à cet engagement humaniste au sens plein du terme tel que le définit donc l'étude de l'humanisme du Quattrocento, je ne peux me contenter d'une perspective très (ou trop) idéaliste. Bien sûr le XV siècle «ignor[ait] l'industrie et l'avènement des masses» ${ }^{31}$ et c'est cela qui change de façon très concrète le projet «d'humanisation» de l'homme que visait la conjonction, et non l'opposition, entre «l'idéal rationnel, l'éducation d'un peuple et la vocation intellectuelle» ${ }^{32}$. Tandis que l'humanisme renaissant prônait, par l'usage d'une certaine «raison», l'apprentissage de la liberté3 ${ }^{33}$, le philanthropisme, humanisme abâtardi, déplace son champ d'application dans le cadre d'une production socialement utile, même si quelques révolutionnaires néohumanistes continuaient et continuent de voir dans les disciplines antiques «le paradoxe de la liberté moderne» ${ }^{34}$. Il est manifeste que le problème s'est dramatisé cette dernière décennie et que cela mérite quelques remarques.

La démocratisation et la massification des enseignements supérieurs ont rendu en effet plus crucial car plus général, le critère de l'employabilité des jeunes sortis du système $^{35}$. Il est le facteur essentiel qui put justifier l'abandon progressif, puis radical des disciplines classiques, «tandis que l'humanité européenne plie devant l'utilitarisme des marchés» ${ }^{36}$. Oui, les enseignements humanistes sont aujourd'hui dénoncés pour leur «incompétitivité» (à l'heure où dominent les pôles de compétence), leur «élitisme» (de combien de spécialistes de Montaigne, Ronsard ou Rabelais, la société actuelle a-t-elle besoin?), leur ethnocentrisme (à l'heure de la mondialisation) ou leur anthropocentrisme (à l'heure de l'universalité du discours scientifique). Et bien que je partage de toute mon âme la révolte de S. Toussaint, profondément convaincue de la nécessité d'une «humanisation par l'étude» ${ }^{37}$, je dois reconnaître avoir «plié» sous le joug de la marchandisation quand est apparue pour moi en tant que doyen d'une faculté de Lettres (directrice d'une Unité de Formation et de Recherche, pour rester fidèle à la modernité) l'évidence des difficultés rencontrées par la majorité des étudiants licenciés quand ceux-ci ne pouvaient choisir la voie traditionnelle des concours de recrutement de notre maison-mère. Il ne s'agissait pas de remettre en cause le nécessaire épanouissement de ceux qui, plus performants, plus libres économiquement aussi, pourraient envisager de conduire leurs études à leur terme (en l'occurrence

taphore empruntée à Max Weber, de la guerre du commerce et de la science, hantaient déjà la révolution néohumaniste».

(31) S. Toussaint, op. cit., p. 102.

(32) S. ToussainT, op. cit., p. 102.

(33) Cf. Montaigne, Les Essais, Livre II, chap. X, Paris, Gallimard, 1962, coll. La Pléiade, p. 388 : «Si j'étudie, je n'y cherche que la science qui traite de la connaissance de moi-même et qui m'instruise à bien mourir et à bien vivre».

(34) S. TOussainT, op. cit., p. 75.

(35) S. Toussaint, op. cit., p. 173.
(36) S. Toussaint, op. cit., p. 175. En cette rentrée 2008, les effectifs de l'UFR des Lettres et Sciences humaines que je dirigeais jusqu'en juillet 2008 a encore baissé de près de $10 \%$, baisse qui se confirme d'année en année depuis les quatre dernières années et qui se généralise sur tout l'Hexagone. En revanche, explosent les effectifs en sciences économiques, gestion, et «information et communication» (nouvelles technologies où le contenant compte plus que le contenu).

(37) S. TOUSSAINT, op. cit., p. 175 
pouvoir s'insérer socialement avec pour bagage l'écriture ou la culture classique). Si l'anecdote de Eugenio Garin ${ }^{38}$, heureux que son cordonnier, ancien élève, ait encore conservé à portée de main son Hérodote nous rappelle qu'un enseignant digne de ce nom doit rester un «illuminateur», il demeure que la société actuelle nous impose sans doute de concilier dans nos manières d'enseigner (et non en supprimant nos enseignements) des perspectives qui prennent en compte l'ensemble des données techniques de notre monde sophistiqué. La professionnalisation des enseignements qui s'impose comme une voie qu'on ne peut plus dédaigner, doit être pensée, non pas simplement au service du marché mais au service de ceux qui devront en vivre. Or penser la vie, c'est la penser dans toutes ses dimensions, et les visées éthiques, politiques et métaphysiques qu'une érudition bien sentie véhicule, doivent être maintenues comme dimension primordiale d'une éducation effective. On se prend alors à rêver de la lumière transmise par Ficin quand celui-ci définit comment «humaniser l'âme et hominiser l'univers [ce qui] correspond à l'opération orphique de l'esprit humain, souvent comparé à une lyre cosmique. L'homme se civilise [ainsi] sur le plan d'une sagesse universelle qui lui dévoile l'ordre du monde en sa divinité» ${ }^{39}$. La rigueur et l'intransigeance de l'analyse de Stéphane Toussaint qui dissèque tous les présupposés, toutes les idées de «l'arrière boutique» de nos dirigeants, tous soumis aux lois du marché, ne laissent aucun doute sur la stratégie, programmée, de cette profonde déconstruction de l'«bumanitas» qui scande notre quotidien, national et international. Et l'argument selon lequel, grâce à la démocratisation de l'enseignement supérieur, pourront se réduire les inégalités sociales s'effondre à la moindre statistique car, comme Gramsci le prévoyait, il y a plus de soixante ans, «l'aspect le plus paradoxal de ce nouveau type d'école [professionnelle] est qu'il passe et qu'il est tenu pour démocratique, alors qu'il est non seulement destiné à perpétuer les différences sociales mais aussi à les cristalliser en des formes chinoises $)^{40}$. Le problème est donc posé et nous invite, à la lumière du parcours initié par l'ouvrage de Stéphane Toussaint, tout autant scientifique que politique, à nous interroger sur la manière d'en trouver sinon l'issue, du moins celle qui, dans cette période de crise, renouera les fils d'une transmission du savoir conforme à l'idée qu'on peut et doit se faire de l'bumanitas.

Bien sûr, la «disparition du sujet» dans les nouveaux processus éducatifs est manifeste et «aucun antivol n'est plus donné contre le vol du moi, depuis que s'est produite la fusion suspecte entre la liberté démocratique et la liberté financière» ${ }^{41}$. Mais la condamnation à un chômage irréductible dans une société qui n'offre de salut qu'à ceux qui peuvent «quantifier» leur savoir est une forme encore plus insoutenable du «vol du moi» car elle touche les besoins élémentaires de l'homme, le vivre et le couvert! Le rêve que je caressais et caresse encore, réside dans l'association des deux modes de fonctionnement: conciliation «pragmatique» qui permette à l'étudiant de «diriger sa vie, choisir son métier» mais maintien, voire développement, de l'apprentissage de la maîtrise de «l'outil d'affranchissement dont [ils] disposent initialement par l'étude» ${ }^{42}$. Déjà, dans certains domaines des services publics, se mesure de façon récurrente la carence des réponses humanistes que posent les problématiques bioéthiques ou tout simplement éthiques dans chacun des domaines de la vie (sciences, médecine, droit). En sciences humaines, lorsque les premières générations du «tout commercial» auront pris les rênes des sociétés et institutions de demain, si ce n'est

(38) S. Toussaint, op. cit., p. 236.

(39) S. ToussainT, op. cit., p. 150. La critique est sévère mais la logique réelle lorsque l'auteur reproche aux «pédagogistes» modernes d'être ces «libérateurs officiels de l'enfance [qui] peuvent cacher de redoutables saboteurs de liberté» (p. 177).
(40) Antonio Gramsci, Gli intellettuali, cité et traduit par S. ToussaINT, op. cit., p. 178, le texte italien est donné en note 14 .

(41) S. ToussainT, op. cit., p. 178

(42) S. Toussaint, op. cit., p. 180. 
déjà fait, il sera trop tard pour ressusciter une culture éradiquée de tout fondement historique et métaphysique. Les réformes des «masters professionnels» initiées par le gouvernement français actuel dans le domaine de l'éducation sont un signal d'alarme qui doit nous inciter, non pas à demeurer dans un statu quo stérile (puisque le ver est déjà dans le fruit) mais à retrouver, dans ce modèle ficinien, les ingrédients d'une culture profonde en ses racines, ouverte en ses sources et active dans son effet direct sur la conscience morale, politique et métaphysique d'une jeunesse en quête d'identification.

Peut-être le salut réside-t-il encore dans l'élaboration au sein des éducations primaire, secondaire, supérieure en Europe, d'un nouveau code de conduite des futurs enseignants qui, sans revenir à l'utopique (et d'ailleurs controversée) fonction de «transmission essentielle de maître à disciple, modèle cher à la plupart des vieux lettrés» ${ }^{43}$, permettrait que soit retrouvée la mission d'éducation qui fonde, sur l'expression d'une sagesse adulte, l'humanisation des nouvelles générations ${ }^{44}$. Cela suppose une réflexion globale sur la fonction éducative, même dans le supérieur, qui définisse autant les «savoirs» à transmettre que les «connaissances» à initier et, pourquoi pas, les «compétences», puisque le mot est devenu essentiel, qui en découlent comme la simplicité humaine, la libre disposition critique, l'intégration dans le vaste «corps de l'humanité». Déjà Rabelais et Montaigne dénonçaient l'étude quand celle-ci se réduisait à un pédantisme où l'«avoir» l'emportait sur l'enrichissement souhaité de l'humain $^{45}$. Rien de pire que le divorce entre «la praxis et la theoria (res et verbum), à l'inverse de la rhétorique et la dialectique renaissantes qui avaient réuni ces moitiés complémentaires» ${ }^{46}$ ! Â nous de conserver aux mots «culture» et «bumanitas» un sens tout autre que celui qui les cantonne à ne faire entendre que l'aspect le plus sclérosé de leur réalité. Il suffit de se souvenir de Pier Paolo Vergerio ${ }^{47}:$ «Puisque l'homme vit dans le risque constant de se voir dérobé à lui-même, le bon maître enseigne au disciple à se défendre contre les voleurs du moi» ${ }^{48}$.

Oui, l'ensemble du discours d'Humanismes et Antibumanismes, de Ficin à Heidegger (et jusqu'à aujourd'hui) est plus qu'une invocation. Par l'abondance et la qualité des analyses, par sa force provocatrice, il nous renvoie, nous lettrés épris d'un humanisme «authentique» (selon la grille de lecture de l'auteur), à nos responsabilités, sans nous offrir l'once d'une excuse qui associerait nos actes de démission

(43) S. Toussaint, op. cit., p. 176.

(44) Cf. Montaigne, Les Essais, Livre I, chap. XXV, éd. cit., p. 137. «Quand bien nous pourrions estre sçavans du sçavoir d'autruy, au moins sages ne pouvons nous estre que de nostre propre sagesse».

(45) Cf. Montaigne, Les Essais, I, XXVI, éd. cit., pp. 135-136: «Tout ainsi que les oyseaux vont quelques fois à la queste du grein et le portent au bec sans le taster pour en faire béchée à leurs petits, ainsi nos pedantes vont pillotant la science dans les livres, et ne la logent qu'au bout de leurs lèvres pour la dégorger seulement et mettre au vent. [...] Mais qui pis est, leurs escholiers et leurs petits ne s'en nourrissent et alimentent non plus; ainsi elle passe de main en main, pour cette seule fin d'en faire parade, d'en entretenir autruy et d'en faire des contes comme une vaine monnoye inutile à tout autre usage et emploite qu'à compter et jetter». Le critère de la critique choisi par Montaigne est bien celui de l' «utilité» sociale; la dénonciation était déjà manifeste p. 137: «Voyez le revenir de là, apres quinze ou seize ans employez: il n'est rien si mal propre à mettre en besongne. Tout ce que vous y recognoissez d'avantage, c'est que son Latin et son Grec l'ont rendu plus fier et plus outrecuidé qu'il n'estoit parti de la maison. Il en devait rapporter l'ame pleine, il ne la rapporte que bouffie». S. Toussaint ne parle pas autrement dans la partie II, chap. 2, p. 176: «Ce faisant, les éducateurs, pédagogues, sociologues anthropologues, philosophes, qui répondent douteusement au nom d'humanistes collaborent à synchroniser le déni théorique avec le déni pratique en soutenant la flexibilité, la compétitivité, l'employabilité. S'en doutent-ils ou non, leur adhésion hausse d'un cran l'emprise d'un pouvoir situé dans l'avoir pur. À moins bien sûr que ce pouvoir ne se situe désormais dans la vénalité, si l'on définit cette dernière par la mise en vente de biens "gratuits", comme l'érudition, la connaissance et la culture, sur le mode vénal des titres de l'Ancien Régime».

(46) S. ToussainT, op. cit., p. 89.

(47) S. ToussainT, op. cit., p. 132.

(48) S. Toussaint, op. cit., p. 133. 
à l'expression libérale de «bons sentiments». Quand Stéphane Toussaint conclut: «l'histoire de la vocation semble s'être arrêtée, mais elle pourrait reprendre» ${ }^{49}$, l'invitation est manifeste. Plus que jamais l'enseignement des grands textes humanistes de la Renaissance est vital. À nous de nous réinvestir dans le système éducatif pour que, conscients de toutes les manipulations subies, nous puissions appliquer dans les faits, les richesses et valeurs des connaissances dont nous défendons plus que la survie, la vie, d'autant que l'enjeu est autant politique que scientifique. Il est plus qu'opportun de conclure avec Stéphane Toussaint sur les théories, toujours actuelles, de Niethammer: «En affirmant que le mépris politique de l'érudition conduit à la mort de ce qu'il nomme, ailleurs et à l'instar de Kant, le cosmopolitisme, Niethammer profère moins une menace culturelle qu'il ne formule un jugement sur la violence engendrée par 1789, comme Edmund Burke en avait déjà donné l'exemple aux Allemands. Il n'en demeure pas moins que des paroles aussi prophétiques chargent les nations modernes d'une responsabilité nouvelle, celle de prévenir la barbarie par un humanisme politique, fondé sur l'impératif de gouverner avec les doctes et les savants. Gouverner contre eux ou appuyer un gouvernement sur le mépris des sciences, équivaudrait inversement à un vandalisme politique, châtié par une chute rapide dans la barbarie. Ce qui se profile, dans cet avertissement aux politiciens, insouciant du péril barbare, pourrait s'appeler l'incorporation de la barbarie, puisque son foyer ne réside plus, comme les Athéniens de Périclès, auprès de populations allogènes et allophones, ou comme les Florentins de Vasari, dans des âges ténébreux et gothiques, mais au centre même du pouvoir moderne» ${ }^{50}$. Dont acte.

MYRIAM JACQUEMIER 